\title{
Realization of Fast Fourier AC Sampling Algorithm Applied to Mine High Voltage Grid Parameter Detection
}

\author{
Liu Zhongfu, Li Shaomin and Yang Yaning \\ College of Information \& Communication Engineering \\ Dalian Nationalities University, Liaoning Dalian, China \\ lzhongfu@163.com
}

\begin{abstract}
In the mine protection of high voltage power grid, quick and accurate detection of network parameters and power quality parameters is significant for the reliability, security and continuity of the power supply system. Fast AC sampling algorithm with low computational complexity and high precision can obtain a variety of electrical parameters accurately, to guarantee real-time system monitoring and protection. In this paper, in-depth study on short-circuit protection and overload protection works of the mine high voltage power grid is undertaken, the advantages and disadvantages of Fourier algorithm and root mean square (RMS) algorithm in AC sampling algorithm as well as the application conditions are analyzed. On this basis, a fast Fourier AC sampling algorithm applied to mine high voltage grid parameters detection is proposed, and the algorithm is analyzed; at the same time, hardware circuit of mine high voltage power protection system and related software are designed based on Fast Fourier AC sampling algorithm, and the system is verified by experiments. Experimental results show that the system based on fast Fourier AC sampling algorithm is stable, and network parameter detection is accurate, with certain application value.
\end{abstract}

Keywords: Fast Fourier algorithm, sampling, mine high voltage grid, short-circuit protection

\section{Introduction}

With the increase of the modernization degree of coalmine and the increase of the underground high-voltage power supply distance, the reliability, safety and continuity of the coalmine power system presents increasingly high demand. The impacts and imbalances of mine nonlinear loads lead to the deterioration of power supply quality, and network parameters and power quality parameters become important indicators of controlling power supply line status and evaluating quality of power supply. Accurate grid data collection, fault diagnosis and data processing have become the focuses of normal run of power.

Existing power grid parameter monitoring systems are restricted by devices and analysis methods, most of which cannot capture the transient and short-term disturbance information in the system quickly and accurately. How to acquire each analog power in the system accurately and quickly determines whether the network can be normally operated.

Sampling algorithm accuracy and timeliness contradict with each other, so how to achieve a reasonable balance between computational accuracy and speed of the algorithm is the key of protecting algorithm research by grid parameter monitoring.

In this paper, the principles of short-circuit protection and overload protection in the mine high voltage power grid are studied deeply, and on this basis, practical fast Fourier AC sampling algorithm is proposed, which can guarantee speed and accuracy at the same time, implementation plan of software and hardware in mine high voltage grid monitoring 
system is given, and the system is tested. Test results show that the sampling algorithm accuracy and real-time performance can meet the standards, and various technical indicators of the system meet the design requirements.

\section{Protection Principle of Mine High Voltage Grid}

\subsection{Principle of Short-Circuit Protection}

In mine high voltage grid protection, current fast-tripping protection should be applied to various short-circuit faults. During normal operation of the grid, the load current passes through the transmission line; in interphase short circuit, short-circuit current passes, which is generally much larger than the load current. Therefore, short-circuit protection usually applies current sensing principle, which is to take the magnitude of the current as criterion, to select the size of the operating current according to the size of the passing current through the protection system during short circuit to control the scope of the protection system by the size of the operating current. But this kind of criterion is sometimes not reliable enough, because it may result in a "dead zone" of protection[1].

The circuit protection diagram of high voltage power supply system is shown in Figure 1 , where T is power transformer, $S W_{1}, S W_{2}$ and $S W_{3}$ are circuit breakers of each branch, and $M_{1}$ and $M_{2}$ are motors. The cross-sectional area of cable from transformer to $d_{2}$ is small, and the distance is long; the cross-sectional area of cable from transformer to $d_{1}$ is large, and the distance is short. The minimum two-phase short-circuit current at $d_{2}$ when short-circuit failure occurs is $I_{d 2 \text { min }}$, which may be less than the maximum starting current $I_{d 1 q}$ at $d_{1}$, namely

$$
I_{d 2 \min }<I_{d 1 q}
$$

In order to distinguish the starting current from short-circuit current under the conditions above, and guarantee the sufficiently high sensitivity when short circuit occurs in the farthest end of the system, "phase-sensitive protection" principle is adopted in the system.

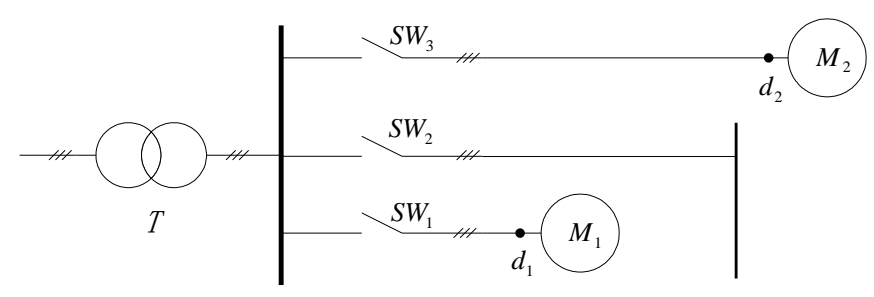

\section{Figure 1. Schematic Diagram of "Dead Zone" in the Short Circuit Protection For 6kv Power Distribution System}

The basic starting point of "phase-sensitive protection" is to detect both the size of the short-circuit current and the impedance angle of the short circuit, and the two phases present a coordinative relation. In general, the power factor at the starting time is small, and the power factor of the short circuit is relatively high. Based one this principle, it is very easy to distinguish starting current and short-circuit current by detecting the phase lag $\varphi$ between the current and the voltage in software. However, phase-sensitive protection is not completely reliable; if short circuit occurs at the exit of transformer, as the transformer resistance $R_{b}$ is small, its impedance is mainly manifested by the inductor $X_{b}$, namely 


$$
X b>>R b
$$

Then the power factor in short circuit at the exit of the transformer is very small, typically less than 0.2 , and there is a certain "dead zone" in phase-sensitive protection. To eliminate this shortcoming, another additional measure needs to be taken: when the current is particularly large, short circuit protection should be started, regardless of the phase angle.

Through the above analysis, it can be known phase-sensitive protection improves the reliability of action based on two electrical parameters as the sample size. Especially when short-circuit current is small and the power factor is large, the operation is more accurate. Combined with additional criteria, the identification of short-circuit fault is made more accurate and reliable.

\subsection{Working Principle of Overload Protection}

Overload is an abnormal running state; the overload of a certain range or certain period of time is allowed, but long-term overload is not allowed. Prolonged overload can cause heat accumulation of the cable, the electrical insulation level will gradually decline, eventually leading to the break of insulation, and the damage of equipment. Heat accumulation is related to two factors, current and time.

Identification method of overload adopts the current sampling principle, and overload current is generally smaller than short-circuit current.

Overload protection is realized by using inverse-time operation characteristics; the higher the overload multiple $\beta$ (actual current/rated operating current, $\mathrm{Ig} / \mathrm{Ie}$ ) is, the shorter overload time it is allowed.

Overload inverse-time characteristics are related to the actual overload features of the equipment and the line; overload inverse-time curve is shown in Figure 2.

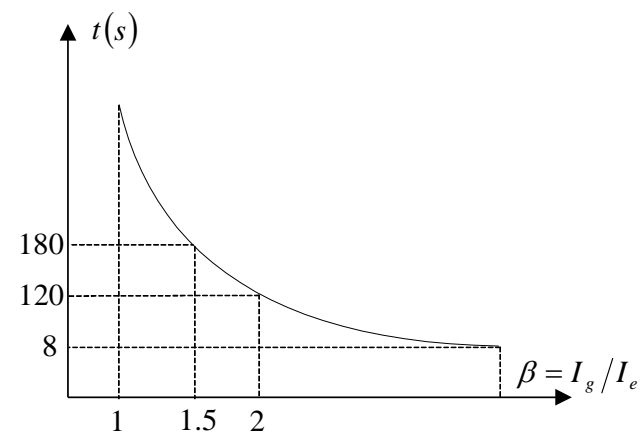

Figure 2. Inverse-Time Curve of Overload

\section{Fast Fourier AC Sampling Algorithm}

\subsection{Analysis of AC Sampling Algorithm}

AC sampling algorithm is applied widely; there are many different algorithms according to different conditions, and it can be divided into sinusoidal model algorithm and non-sinusoidal cycle model algorithm according to its model function, in which the sinusoidal model algorithm mainly includes maximum algorithm, single-point algorithm, half-cycle integration sampling two-point sampling, while non-sinusoidal model algorithm includes RMS algorithm and Fourier algorithm [2].

Each algorithm has both advantages and disadvantages, applied in power system differentially. Sinusoidal model algorithm is based on the ideal sampling values that are provided for the raw data of the algorithm as pure sinusoidal quantity. Actually current 
and voltage in the system have various harmonic components; after the fault, current and voltage both contain a variety of transient components, and various errors may be introduced into the data acquisition system as well, namely, the actual sampled signal is not pure sinusoidal quantity, so non-sinusoidal cycle model algorithm is analyzed, which means Fourier algorithm and RMS algorithm.

RMS algorithm applies only to a sinusoidal signal, and the algorithm itself has not filtering effect, by which the calculation is simple and fast, with high calculation accuracy when there are more sampling points.

Fourier transform algorithm is applicable to sinusoidal or non-sinusoidal periodic signals, not influenced by power symmetry, which is applied widely, with filtering function. It can not only filter all integer harmonic components and pure DC components completely, but also have inhibition effect on non-integer high-frequency component and the low-frequency component included in attenuated non-periodic components, which can give the signal of each subharmonic, with high calculation precision, and conducive to harmonic analysis, but the speed is not fast enough[3].

\subsection{Fast Fourier AC Sampling Algorithm}

Full-wave Fourier algorithm itself has good filtering performance, which can filter out all the DC components and integer harmonic components, and can also inhibit the highfrequency component to some extent, but this algorithm is proposed based on the model of periodic function, and this principle itself restricts the ability of the Fourier algorithm to deal with the decaying $D C$ component prevalent in relay system failure. Improved Fourier algorithm mainly focuses on how to improve the mathematical model of the Fourier algorithm, in which the decaying $D C$ component is also taken as part of a mathematical model to explore a method to filter decaying $D C$ component more accurately [4].

The algorithm starts entirely from discrete sample values, in which continuous function integrals do not appear, so that the exact solution is derived [5].

Current signal model is set as

$$
\begin{gathered}
i(t)=A e^{-\alpha t}+\sum_{n=1}^{M} I_{m n} \sin \left(n \omega t+\varphi_{n}\right) \\
=A e^{-\alpha t}+\sum_{n=1}^{M}\left(I_{m n} \sin \varphi_{n} \cos n \omega t+I_{m n} \cos \varphi_{n} \sin n \omega t\right) \\
\text { Making }\left\{\begin{array}{l}
I_{R n}=I_{m n} \sin \varphi_{n} \\
I_{I n}=I_{m n} \cos \varphi_{n}
\end{array}\right.
\end{gathered}
$$

Then $i(t)=A e^{-\alpha t}+\sum_{n=1}^{M}\left(I_{R n} \cos n \omega t+I_{I n} \sin n \omega t\right)$, and a period is selected randomly from above models to conduct $\mathrm{N}$-equal-portion sampling, and the expression of discrete sampling points are

$$
i(k)=A e^{-\alpha \frac{T}{N} k}+\sum_{n=1}^{M}\left[I_{R n} \cos \left(n \frac{2 \pi}{N} k\right)+I_{I n} \sin \left(n \frac{2 \pi}{N} k\right)\right]
$$

The equation above is subject to Discrete Fourier Transform to obtain: 


$$
\begin{array}{r}
I_{R n}^{*}=\frac{2}{N} \sum_{k=0}^{N-1}\left[A e^{-\alpha \frac{T}{N} k} \cos \left(n \frac{2 \pi}{N} k\right)\right]+I_{R n} \\
I_{I n}^{*}=\frac{2}{N} \sum_{k=0}^{N-1}\left[A e^{-\alpha \frac{T}{N} k} \sin \left(n \frac{2 \pi}{N} k\right)\right]+I_{I n} \\
\text { Making } \Delta I_{R n}=\frac{2}{N} \sum_{k=0}^{N-1}\left[A e^{-\alpha \frac{T}{N} k} \cos \left(n \frac{2 \pi}{N} k\right)\right] \\
\Delta I_{I n}=\frac{2}{N} \sum_{k=0}^{N-1}\left[A e^{-\alpha \frac{T}{N} k} \sin \left(n \frac{2 \pi}{N} k\right)\right]
\end{array}
$$

then it can be seen $I_{R n}=I_{R n}^{*}-\Delta I_{R n}$

$$
I_{I n}=I_{I n}^{*}-\Delta I_{I n}
$$

From 10 and 11, it can be seen that if A and $a$ can be computed, $I_{R n}$ and $I_{I n}$ can be calculated accurately through 8, 9, 10 and 11 .

The method to calculate $\mathrm{A}$ and $a$ is derived as follows.

$$
\begin{gathered}
\sum_{k=0}^{N-1} i(k)=\sum_{k=0}^{N-1}\left(A e^{-\alpha \frac{T}{N} k}\right)+\sum_{k=0}^{N-1}\left[I_{R n} \sum_{k=0}^{N-1} \cos \left(n \frac{2 \pi}{N} k\right)+I_{I n} \sum_{k=0}^{N-1} \sin \left(n \frac{2 \pi}{N} k\right)\right] \\
=\sum_{k=0}^{N-1}\left(A e^{-\alpha \frac{T}{N} k}\right)=A \frac{1-e^{-\alpha T}}{1-e^{-\alpha \frac{T}{N}}} \\
i(0)-i(N)=A\left(1-e^{-\alpha T}\right) \\
\text { Making } \sum_{k=0}^{N-1} i(k)=B \\
i(0)-i(N)=C \\
\text { Then } B=A \frac{1-e^{-\alpha T}}{1-e^{-\alpha \frac{T}{N}}} \\
C=A\left(1-e^{-\alpha T}\right)
\end{gathered}
$$

Therefore,

$$
\alpha=\frac{N}{T} \ln \left(\frac{B}{B-C}\right)
$$




$$
A=\frac{C}{1-\left(\frac{B}{B-C}\right)^{N}}
$$

Because As 18 contains logarithm, not convenient for the application in practical engineering, it is simplified.

Based on 17 and 16,

$$
\frac{C}{B}=1-e^{-\alpha \frac{T}{N}}
$$

Generally, $T=0.02 s$, and if the time constant of decaying aperiodic component is $0.02 s$, then $\alpha=50$. Making $N=12$, and $(\alpha T / N)=1 / 12$, it can be seen in the condition of not small time constant of decaying aperiodic component, Taylor series expansion is conducted to $e^{-\alpha T / N}$, and the first two items are taken to meet the accuracy requirements, as in 19 and 20.

$$
\begin{aligned}
& e^{-\alpha \frac{T}{N}}=1-\alpha \frac{T}{N}+\frac{1}{2}\left(\alpha \frac{T}{N}\right)=D \\
& \frac{C}{B}=\alpha \frac{T}{N}-\frac{1}{2}\left(\alpha \frac{T}{N}\right)^{2}
\end{aligned}
$$

It can be obtained that

$$
\begin{gathered}
\alpha=\frac{2 N \frac{C}{B}}{T\left(1+\sqrt{1-2 \frac{C}{B}}\right)} \\
A=\frac{C}{1-\left(\frac{B-C}{B}\right)^{2}}
\end{gathered}
$$

$\alpha$ is not necessary to be solved in practical engineering, and instead, $\alpha T / N$ is to be solved; it can be seen from 23

$$
\begin{array}{r}
\alpha \frac{T}{N}=\frac{2 \frac{C}{B}}{1+\sqrt{1-2 \frac{C}{B}}}, \text { so it can be obtained from } 22 \text { that } \\
D=e^{-\alpha \frac{T}{N}}=1-\alpha \frac{T}{N}+\frac{1}{2}\left(\alpha \frac{T}{N}\right)^{2}
\end{array}
$$

8 and 9 can be rewritten as

$$
\begin{aligned}
& \Delta I_{R n}=\frac{2}{N} A D^{k} \cos \left(n \frac{2 \pi}{N} k\right) \\
& \Delta I_{I n}=\frac{2}{N} A D^{k} \sin \left(n \frac{2 \pi}{N} k\right)
\end{aligned}
$$


$6,7,26$ and 27 are substituted into 10 and 11 , and then it can be obtained:

$$
\begin{aligned}
& I_{R n}=\frac{2}{N} \sum_{k=0}^{N-1}\left(i(k)-A D^{k}\right) \cos \left(n \frac{2 \pi}{N} k\right) \\
& I_{I n}=\frac{2}{N} \sum_{k=0}^{N-1}\left(i(k)-A D^{k}\right) \sin \left(n \frac{2 \pi}{N} k\right)
\end{aligned}
$$

Therefore, after the calculation of A and D previously, the original sample sequence is corrected and then $I_{R n}$ and $I_{I n}$ can be obtained by another full-wave Fourier transform.

The effective value $I_{m n}$ and the initial phase $\varphi_{n}$ of the signal are solved.

$$
\begin{aligned}
& I_{m n}=\sqrt{I_{R n}^{2}+I_{I n}^{2}} \\
& \varphi_{n}=\arctan I_{I n} / I_{R n}
\end{aligned}
$$

\section{Hardware Design of Mine High Voltage Parameter Detection System}

The hardware circuit principle diagram of computer-based high-voltage grid parameter detection system is shown in Figure 3. The whole system consists of the following components:

(1) Computer control unit: for the realization of data collection, calculation, logic judgment, timing and other functions.

(2) Analog input unit: for collecting and computing the voltages of A, B and C as well as the currents of $\mathrm{A}$ and $\mathrm{C}$ and zero-sequence voltage.

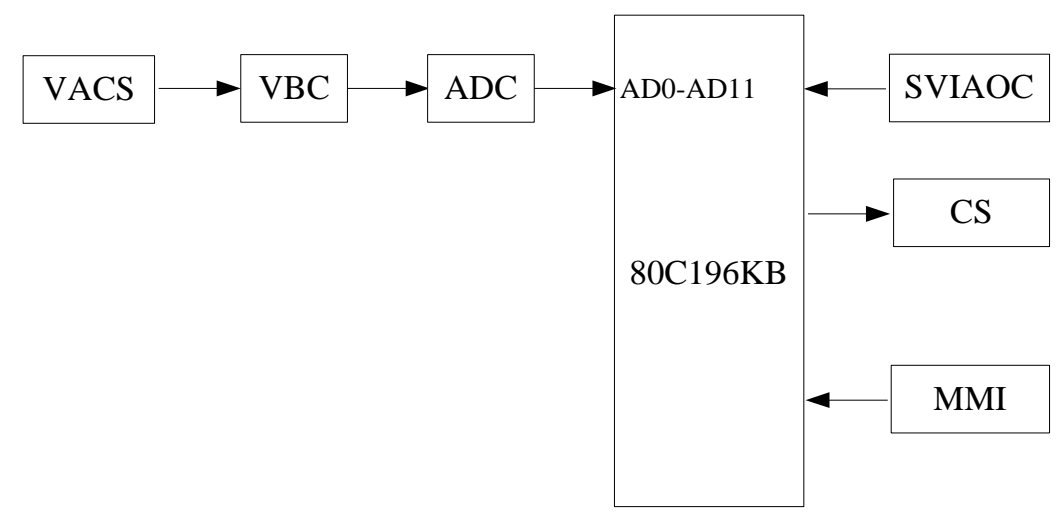

Figure 3. Diagram of Grid Parameter Detection System

As in Figure 3, VACS is Voltage and Current Sampling, $V B C$ is Voltage Bootstrap Circuit, $A D C$ is AD Converter, SVIAOC is Switch Variable Input and Output Circuit, $C S$ is Communication Schematic and $M M I$ is Man-machine Interface.

\subsection{Computer Control Unit}

Computer control unit is the core of the monitoring and control system, which is in charge of data collection, calculation, logic judgment, timing, storage and so on. In the design of microcontroller application system, the first thing to consider is the selection of CPU. 80C196KB microcontroller with high performance and low cost is adopted in the system, which can meet the requirements of real-time performance, easy for upgrading. 


\subsection{Analog Input Unit}

Analog input is very important for high-voltage grid parameter detection system; the system captures physical parameters of the system properly to determine the current operating condition of the grid by calculation, so as to send the correct control information. In the design, AC sampling technology is applied, and the principle block diagram of the analog input is shown in Figure 4, where $V B C$ is Voltage Bootstrap Circuit, $L P F C$ is Low-pass Filter Circuit, $S A H C$ is Sampling And Holding Circuit, $A M$ is Analog Multiplexer and $A D C$ is AD Converter.

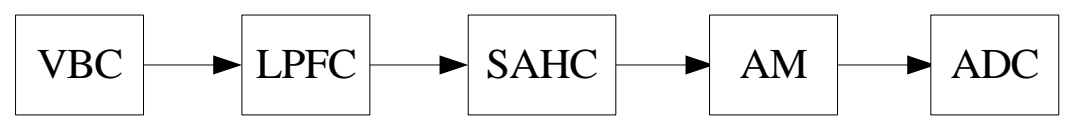

Figure 4. Block Diagram Of Input Unit For Analog Signals

4.2.1. Voltage Bootstrap Circuit: The performance of voltage and current transformers directly affects the accuracy of signal acquisition, related to the reliability and stability of the whole system, so the circuit design of the voltage bootstrap circuit is crucial.

Primary current transformer converts high current into low current and high-voltage circuit and isolates high-voltage circuit from low-voltage circuit, so that there is no direct link in electricity between them. In the rated condition, primary current transformer outputs the current of 5A, secondary current transformer outputs primary current of 5A, so as to standardize and serialize the designs of protection systems and secondary-circuit equipment. In the condition of small current of secondary-circuit device, the cost is low, and easy to maintain, and in addition, it can ensure the safety of the operator. Similarly, voltage transformer converts the high-voltage signal into low-voltage one, to isolate the high-voltage from low-voltage signal, to ensure safety and reduce the size and cost of the secondary circuit, and secondary voltage and primary voltage of voltage transformer is made proportional, which reflects the primary side change of the system correctly.

The system adopts DVDI001 ultra-small precision universal AC voltage and current transformer produced by Beijing Chuang Si Fang Electronic Co., Ltd.

When DVDI001 transformer is used as voltage transformer, it is actually a currentmode voltage transformer, of which the application circuit is shown in Figure 5:

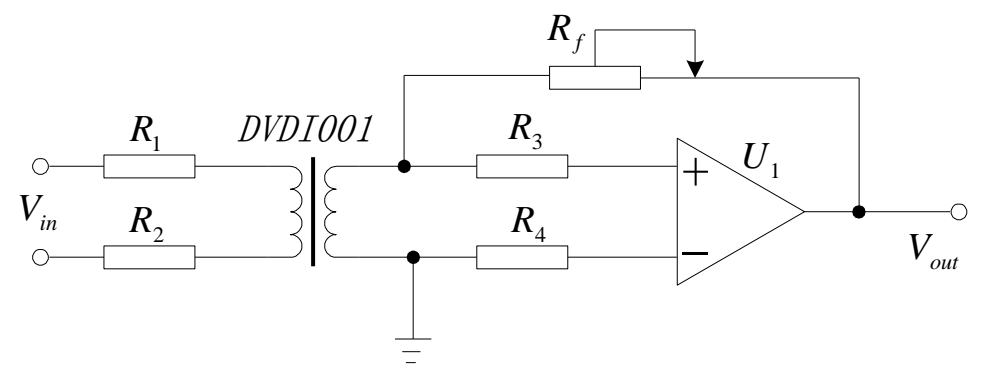

Figure 5. PT Voltage Bootstrap Circuit

4.2.2. Ad Converter: Considering the requirements in $A D$ converter accuracy and speed of the system, $A D 1674$ is chosen for the system, which is 12-bit $A D$ converter with high performance introduced by the United States $A D$ Company.

$A D 1674 J N$ is used in the system, of which the circuit connection with $80 C 196 \mathrm{~KB}$ microcontroller is shown in Figure 6. 
In the figure, $A D 1674$ is bipolar analog signal conversion, $10 \mathrm{~V}_{\text {in }}$ is applied for $\pm 5 \mathrm{~V}$ analog input of the multiplexer, and 1LSB corresponds to $5 \mathrm{mV}$. AD1674 works in an independent manner, $\mathrm{CE}$ and $12 / 8$ are connected with $+5 \mathrm{~V}, /$ cs and $\mathrm{A}_{0}$ are connected with $0 \mathrm{~V}$, and $R / C$ is taken as data read and data conversion control; Y6 of $74 H C 138$ is connected with $R / C$ of $A D 1674$, when $R / C=1$, the converted data appear in the output end, and when $R / C=0$, an A/D conversion is started. STS is connected with P0.4 of the microcontroller; when STS $=1$, it means a conversion is underway, and STS $=0$ indicates $\mathrm{A} / \mathrm{D}$ conversion is completed. The output data can be read out from the output terminal.

As for $80 C 196 K B$ microcontroller, the converted data can be read out once.

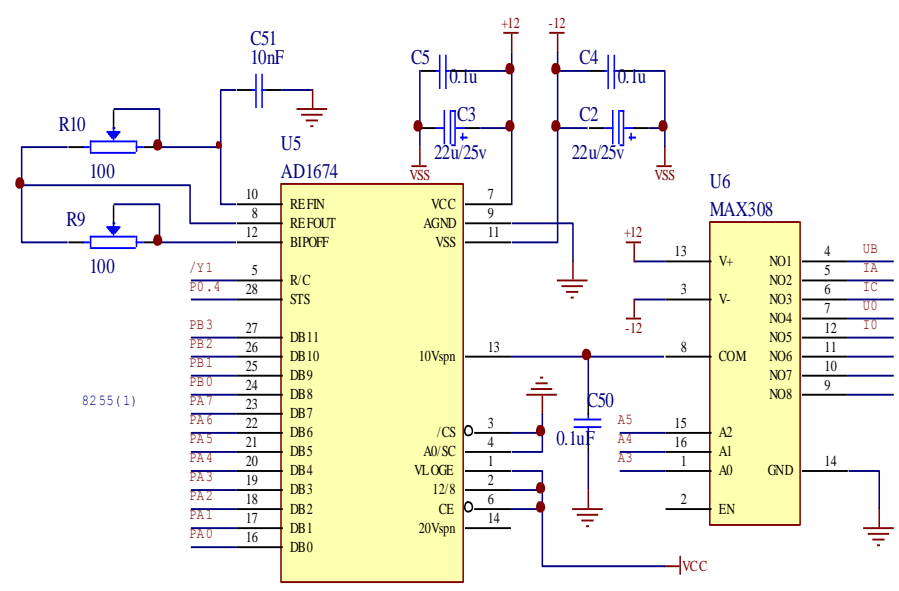

Figure 6. Interface to MAX308 and AD1674

\section{Experimental Verification of the Algorithm}

\subsection{Current Protection Test}

The rated values such as rated operating current, rated short-circuit multiple, rated overload multiple and overload delay time are input by DIP plate. After the system is powered, the output AC current from current booster are joined into $I_{a}$ and $I_{c}$ phases to regulate the input voltage of current booster, to change the size of the current of $I_{a}$ and $I_{c}$ phases. When the current is increased to rated overload value, the protection system trips by different time delay, and meanwhile the LCD shows the cause of the fault "Overload accident!" with the corresponding indicator lightened.

When the current of $I_{a}$ and $I_{c}$ phases reaches the rated short-circuit current of system, "short circuit" indicator is lightened, the cause "Short-circuit fault!" is displayed, and the relay acts instantaneously to make the circuit breaker tripped.

The experimental data of overload and short-circuit protection (IN is assumed to be 200A, and the "third" level is selected for overload delay time) are shown in Table 1.

Table 1. Experimental Data of Overload And Short-Circuit Protection

\begin{tabular}{|c|c|c|c|c|c|}
\hline \multirow[b]{3}{*}{$\begin{array}{l}\text { Ove } \\
\text { rload }\end{array}$} & \multicolumn{2}{|c|}{ Operate value } & \multicolumn{2}{|c|}{ Operate time } & \multirow[b]{2}{*}{$\begin{array}{l}\text { Concl } \\
\text { usion }\end{array}$} \\
\hline & Designed value & Actual value & $\begin{array}{l}\text { Desig } \\
\text { ned } \\
\text { value }\end{array}$ & $\begin{array}{l}\text { Actual } \\
\text { value }\end{array}$ & \\
\hline & $\mathrm{I}_{\mathrm{W}} \leq 0.8 \mathrm{I}_{\mathrm{N}}$ & $150 \mathrm{~A}$ & $8.8 \mathrm{~s}$ & $8.8 \mathrm{~s}$ & $\begin{array}{l}\text { Qualif } \\
\text { ied }\end{array}$ \\
\hline
\end{tabular}




\begin{tabular}{|c|c|c|c|c|c|}
\hline & $0.8 \mathrm{I}_{\mathrm{N}} \leq \mathrm{I}_{\mathrm{W}} \leq 1.0 \mathrm{I}_{\mathrm{N}}$ & $180 \mathrm{~A}$ & $7.0 \mathrm{~s}$ & $7.0 \mathrm{~s}$ & $\begin{array}{l}\text { Qualif } \\
\text { ied }\end{array}$ \\
\hline & $1.0 \mathrm{I}_{\mathrm{N}} \leq \mathrm{I}_{\mathrm{W}} \leq 1.2 \mathrm{I}_{\mathrm{N}}$ & $220 \mathrm{~A}$ & $5.8 \mathrm{~s}$ & $5.8 \mathrm{~s}$ & $\begin{array}{l}\text { Qualif } \\
\text { ied }\end{array}$ \\
\hline & $1.2 \mathrm{I}_{\mathrm{N}} \leq \mathrm{I}_{\mathrm{W}} \leq 1.4 \mathrm{I}_{\mathrm{N}}$ & $260 \mathrm{~A}$ & $5.0 \mathrm{~s}$ & $5.0 \mathrm{~s}$ & $\begin{array}{l}\text { Qualif } \\
\text { ied }\end{array}$ \\
\hline $\begin{array}{l}\text { Sho } \\
\mathrm{rt} \\
\text { circuit }\end{array}$ & $8 \mathrm{I}_{\mathrm{N}} \leq \mathrm{I}_{\mathrm{W}}$ & $1560 \mathrm{~A}$ & $100 \mathrm{~ms}$ & $100 \mathrm{~ms}$ & $\begin{array}{l}\text { Qualif } \\
\text { ied }\end{array}$ \\
\hline
\end{tabular}

\subsection{Over-Voltage and Under-Voltage Experiments}

Input voltages $U_{a}, U_{b}$ and $U_{c}$ are regulated; when the input voltage is more than $118 \%$ of rated voltage, the system takes action, and "Over-voltage fault" is displayed by the system; when the voltage is less than $65 \%$ of rated voltage, the system takes action, and "Under-voltage fault" is displayed by the system. The experimental data of overvoltage and under-voltage protection are shown in Table 2.

Table 5. Experimental Data Of Over-Voltage And Under-Voltage Protection

\begin{tabular}{ccccc}
\hline & $\begin{array}{c}\text { Voltage } \\
\text { level }\end{array}$ & $\begin{array}{c}\text { Designed } \\
\text { operate value }\end{array}$ & $\begin{array}{c}\text { Actual } \\
\text { voltage }\end{array}$ & $\begin{array}{c}\text { Con } \\
\text { clusion }\end{array}$ \\
\hline $\begin{array}{c}\text { Over } \\
\text {-voltage }\end{array}$ & $6 \mathrm{KV}$ & $>7080 \mathrm{~V}$ & $7200 \mathrm{~V}$ & $\begin{array}{c}\text { Qual } \\
\text { ified }\end{array}$ \\
$\begin{array}{c}\text { Unde } \\
\text { r- } \\
\text { voltage }\end{array}$ & $6 \mathrm{KV}$ & $<3900 \mathrm{~V}$ & $3600 \mathrm{~V}$ & $\begin{array}{c}\text { Qual } \\
\text { ified }\end{array}$ \\
\hline
\end{tabular}

It can be seen from the experiment $\mathrm{s}$ of the current protection, leakage protection, surveillance protection, overvoltage protection and undervoltage protection, this system meets the design requirements, of which the experimental data and theoretical data are basically identical.

\section{Conclusion}

The protection principles of short-circuit, overload, overvoltage and undervoltage of mine high-voltage grid are studied in the paper, with a more comprehensive consideration of the grid operation that is changed in real time. On this basis, a fast Fourier AC sampling algorithm is proposed, AC sampling is applied to the secondary information for direct and high-speed sampling, fast Fourier transform algorithm is used to calculate the characteristic quantities of voltage and current in the grid, to identify and eliminate interference, distinguishing and detecting the accidental ones, and then the quantities to be controlled are monitored, analyzed, counted and judged, so that the credibility and the reliability are guaranteed. Experimental results show that the system based on fast Fourier AC sampling algorithm works stably, with accurate detection of network parameters, which is of great value in application. 


\section{References}

[1] A. M. Manilov and D. A. Mel'nik, "A method of increasing the sensitivity of protection from singlephase short-circuits to ground in the 6-10 kV network [J]", Power Technology and Engineering, 2012, vol. 46, no. 2, pp.153-156.

[2] M. Senthil Arumugam and M. V. C. Rao, "On the improved performances of the particle swarm optimization algorithms with adaptive parameters, cross-over operators and root mean square (RMS) variants for computing optimal control of a class of hybrid systems[J]", Appl. Soft Comput., 2008, vol. 8.

[3] S. V. Len'kov, "Measurement of the amplitudes of the harmonics of a periodic signal using a fast Fourier transform algorithm [J]", Measurement Techniques, 2006, vol. 49, no. 2, pp. 173-177.

[4] Erik Hsiao and Seong H. Kim, "Analyzing Periodic Signals in Rotating Pin-on-Disc Tribotest Measurements Using Discrete Fourier Transform Algorithm [J]”, Tribology Letters, 2009, vol. 3, no. 2, pp. 141-147.

[5] Kim Hwi, Yang Byungchoon and Lee Byoungho, "Iterative Fourier transform algorithm with regularization for the optimal design of diffractive optical elements[J]", Optical Society of America. Journal A: Optics, Image Science, and Vision, 2004, vol. 21, no. 12, pp. 2353-65.

\section{Author}

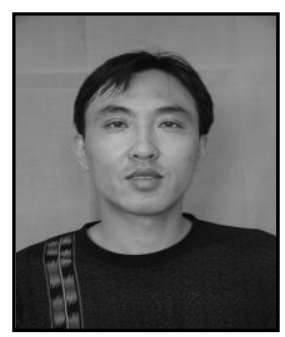

Liu Zhongfu, he was borned in 1973, received the Master degree in Power Electronics and Motor Drives from Taiyuan University of Technology in 2003. Now he is a lecturer at College of Electric Information Engineering, Dalian Nationalities University, China. His papers have been published in some well-known international Journals. His main interests include Embedded System, Technical application of Internet of Things. 
International Journal of Control and Automation Vol.8, No.11 (2015) 